\title{
Re: Training in the use of intrapartum electronic fetal monitoring with cardiotocography: systematic review and meta-analysis. Cardiotocography training is a complex intervention and requires complex evaluations: a letter to editor.
}

\author{
Kate Lightly ${ }^{1}$, Andrew Weeks ${ }^{1}$, and Hazel Scott ${ }^{1}$ \\ ${ }^{1}$ University of Liverpool
}

March 3, 2021

Re: Training in the use of intrapartum electronic fetal monitoring with cardiotocography: systematic review and meta-analysis. Cardiotocography training is a complex intervention and requires complex evaluations: a letter to editor.

Lightly K, Weeks AD, Scott H

Correspondence to Dr Kate Lightly - klightly@liverpool.ac.uk

07875642837

Clinical Research Fellow, Sanyu Research Unit, University of Liverpool and Liverpool Women's NHS Foundation Trust, members of Liverpool Health Partners, Crown Street, Liverpool, L8 7SS.

Professor Andrew D Weeks

Professor of International Maternal Health, Sanyu Research Unit, University of Liverpool and Liverpool Women's NHS Foundation Trust, members of Liverpool Health Partners, Crown Street, Liverpool, L8 7SS.

Professor Hazel Scott

Dean of School of Medicine, School of Medicine, University of Liverpool, Cedar House, Liverpool, L69 3GE.

Running title - CTG training requires complex evaluations

We congratulate Kelly et al on their review on the effects of training in cardiotocography (CTG). ${ }^{\mathrm{i}}$ It is a critical step towards understanding how to correctly implement CTG training. However, we query the relevance of some of the included studies and whether using Kirkpatrick's model adequately captures all of the relevant complexities. We believe that further work is needed to understand how such training will impact on practice.

Some of the CTG research projects reviewed were poorly representative of the needs of clinicians tasked with improving fetal monitoring in their hospitals. For clinicians, their population of interest is practising clinicians who work on labour wards (sometimes infrequently); studies involving undergraduates alone may not be generalisable. Training in intermittent auscultation is also relevant. Considerable detail on the training intervention is required, including not only the format of teaching, but the duration, curriculum and proportion of relevant staff trained. Detail is also required of supporting interventions and context, as training alone is unlikely to impact change. Many would consider 'no training' unethical and therefore not a relevant comparator. In the UK, CTG training and competency is now required for all maternity 
staff. ${ }^{\text {ii }}$ The ultimate aim of CTG (and therefore CTG training) is to detect the hypoxic fetus, so that timely intervention can be undertaken to avoid perinatal harm, without unnecessary intervention. Therefore, the outcomes of interest have to include intrapartum stillbirths, hypoxic ischaemic encephalopathy and mode of delivery.

Kirkpatrick's model was used in this review to evaluate training at four levels (reaction, knowledge, behaviour change and organisational performance). However, it does not attempt to understand why interventions work, or the context, or the causal pathways between training and change in practice. ${ }^{\text {iii }}$ Whether participants like training ('reaction') is of little relevance, and knowledge acquisition ('knowledge') is a proxy which does not equate with improved on the job performance and outcomes. Maternal and perinatal outcomes ('performance') were only collected in a small number of studies.

CTG training is a complex intervention which aims to create change, not simply knowledge acquisition. We therefore believe that a formal Realist Evaluation is needed. This emerging methodology has been used successfully to understand healthcare change processes and supplement traditional Cochrane style reviews. It aims to understand why complex interventions work, how, for whom, in what context and to what extent ${ }^{\text {iv }}$. Collaboration with the relevant authors to gain detailed intervention descriptions, with a realist approach, may add some much needed explanatory power to this critical subject.

Training alone is unlikely to impact change. Even the best educational package will fail without the necessary support - it needs an educational and working culture which supports learning and change, aligned and clear policies, and motivated, well supported drivers and leaders.

How doctors learn and what supports them to put new knowledge into practice are key research questions. High quality, methodologically appropriate, properly funded studies are needed to address these questions. Not answering them means many research findings are redundant, as they simply will not be implemented.

\section{References}

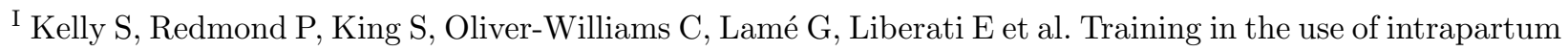
electronic fetal monitoring with cardiotocography: systematic review and meta-analysis. BJOG. 2021; 00: 1-12. https://doi.org/10.1111/1471-0528.16619

ii NHS England. Saving Babies' Lives Version Two. A care bundle for reducing perinatal mortality. London: NHS England 2019. [cited 2021 Feb 3]. Available from: www.england.nhs.uk

iii Moreau KA. Has the new Kirkpatrick generation built a better hammer for our evaluation toolbox? Med Teach. 2017 Sep; 39(9): 999-1001. https://doi.org/10.1080/0142159X.2017.1337874

iv Wong G, Westhorp G, Greenhalgh J, Manzano A, Jagosh J, Greenhalgh T. Quality and reporting standards, resources, training materials and information for realist evaluation: the RAMESES II project. Health Services and Delivery Research. 20175 (28): 1-108. https://doi.org/10.3310/hsdr05280.

Acknowledgements - Nil

Disclosure of interests - Nil

Contribution to authorship

KL wrote the first draft of this letter and then it was revised by AW and HS.

Details of ethics approval - N/A

Funding - Dr Lightly's PhD entitled "Improving intrapartum fetal monitoring in India: A mixed methods approach" is funded by MRC/DfID/Wellcome Trust Joint Global Health Trials Fund. MR/R006/1801 